\title{
Photocarrier generation in metal-free phthalocyanines: Effect of the stacking habit of molecules on the photogeneration efficiency
}

\author{
Yoshihiko Kanemitsu, Aishi Yamamoto, Hiroshi Funada, and Yasuaki Masumoto \\ Institute of Physics, University of Tsukuba, Tsukuba, Ibaraki 305, Japan
}

(Received 24 September 1990; accepted for publication 5 February 1991)

\begin{abstract}
The photocarrier generation mechanism in metal-free phthalocyanines $\left(\mathrm{H}_{2} \mathrm{Pc}\right)$ was studied by time-of-flight photoconductivity measurements. The quantum efficiencies of photogeneration of holes depend on the crystal structure of $\mathrm{H}_{2} \mathrm{Pc}$. The photogeneration efficiencies in $X$ - and $\tau-\mathrm{H}_{2} \mathrm{Pc}$ are high compared with those of $\alpha-$ and $\beta-\mathrm{H}_{2} \mathrm{Pc}$. The lifetimes of the precursor state of free carriers in $X-$ and $\tau-\mathrm{H}_{2} \mathrm{Pc}$ are between those of $\alpha-\mathrm{H}_{2} \mathrm{Pc}$ and $\beta-\mathrm{H}_{2} \mathrm{Pc}$. Moreover, infrared absorption and Raman spectra show that $X$ - and $\tau-\mathrm{H}_{2} \mathrm{Pc}$ are intermediate states between $\alpha-\mathrm{H}_{2} \mathrm{Pc}$ and $\beta-\mathrm{H}_{2} \mathrm{Pc}$. It is pointed out that the photogeneration dynamics of $\mathrm{H}_{2} \mathrm{Pc}$ is controlled by the intermolecular overlap of the $\pi$-electron wavefunctions and the stacking habit of molecules.
\end{abstract}

Recently, numerous organic photoconductive materials having high quantum efficiencies of photocarrier generation have been developed in order to produce high photosensitive photoconductor devices. These are phthalocyanines, squaraines, polyazo materials, polycyclic aromatics, and other compounds. ${ }^{1,2}$ However, the basic photogeneration mechanism in these photoconductors is poorly understood. The understanding of the photogeneration process would reveal a guiding principle for developing new photoconductive materials.

A typical class of high-photosensitive materials, phthalocyanines, has received considerable attention as charge generating materials in electrophotographic receptors and solar cells. Many features of the photogeneration of free charge carriers are explained by the geminate recombination theory of Onsager. ${ }^{3,4}$ The parameters used in the fitting are $Y_{0}$ and $r_{0}$, the initial yield and spatial separation of thermalized electron-hole pairs, respectively. However, the relationship between two parameters and intramolecular and intermolecular properties is not yet clear. ${ }^{5}$ For example, the observed electric field dependence of the photogeneration efficiency shows very large thermalization lengths of the geminate pairs $\left(r_{0}=2-3 \mathrm{~nm}\right){ }^{2,6}$ although the charge transfer (CT) state due to the nearestneighbor electron transfer is a plausible precursor of free charge carriers. ${ }^{7-9}$ The evolution process from an initial nearest-neighbor separation to the much larger thermalized-pair separation and the photogeneration efficiency depend on the intermolecular spacing and the stacking habit of molecules.

In this work, we used metal-free phthalocyanine $\left(\mathrm{H}_{2} \mathrm{Pc}\right)$ as a prototypical organic photoconductor and studied the photocarrier generation in four polymorphic forms of $\mathrm{H}_{2} \mathrm{Pc}$ in order to clarify effects of stacking of molecules on the photogeneration efficiency. It is pointed out that the structure of the nearest-neighbor molecules and the intermolecular overlap of the $\pi$-electron wavefunction determine the photocarrier generation in $\mathrm{H}_{2} \mathrm{Pc}$.

Metal-free phthalocyanine $\left(\mathrm{H}_{2} \mathrm{Pc}\right)$ has been prepared in several polymorphic forms: ${ }^{2} \alpha$ (tetragonal), $\beta$ (face centered monoclinic), $X$ (hexagonal) and recently $\tau . \alpha-, \beta$,
$X$-, and $\tau-\mathrm{H}_{2} \mathrm{Pc}$ characterized by $\mathrm{X}$-ray diffraction were dispersed in a polyester matrix (Vylon 200, Toyobo) purified by repeated reprecipitation. These were coated on an aluminized Mylar sheet to $0.2 \mu \mathrm{m}$ ( 50 wt $\% \mathrm{H}_{2} \mathrm{Pc}$ :polymer) or $5 \mu \mathrm{m}$ ( $20 \mathrm{wt} \% \mathrm{H}_{2} \mathrm{Pc}$ :polymer) thickness. Furthermore, a $0.2 \mu \mathrm{m} \alpha-\mathrm{H}_{2} \mathrm{Pc}$ film was deposited onto the Al Mylar sheet by vacuum evaporation. All $0.2 \mu \mathrm{m}$ films were overcoated with a $10-\mu \mathrm{m}$ charge transport layer (CTL) in order to amplify the transient photocurrent in the time-offlight experiment. The CTL film was a solid solution of $50 \% p$-diethylaminobenzaldehyde-diphenyl hydrazone in polycarbonate. In two-layer samples, a semitransparent gold electrode was finally deposited by vacuum evaporation.

The quantum efficiency of photogeneration of holes, $Y(E)$, is obtained by the potential discharge technique. ${ }^{6}$ In this method, the CTL surface was initially charged by corona, and then discharged by a $0.5-\mu \mathrm{s}, 0.6-\mu \mathrm{m}$ light pulse of $0.2 \mu \mathrm{J} / \mathrm{cm}^{2}$. The temporal change in surface voltage was monitored by using an electrostatic voltmeter and a storage oscilloscope. The quantum efficiency $Y(E)$ is given by, $Y(E)=C \delta V / e N$, where $C$ is the capacitance of the film, $N$ is the absorbed photon number, $e$ is the electric charge, and $\delta V$ is the light-induced voltage drop.

In the delayed-collection-field (DCF) experiment, the two-layer structure films were illuminated through the gold electrode by a $0.6-\mu \mathrm{m}, 0.5-\mu \mathrm{s}$ flash pulse. The light pulse excited in the $\mathrm{H}_{2} \mathrm{Pc}$ layer only in zero field and a high field of $3.5 \times 10^{5} \mathrm{~V} / \mathrm{cm}$ was applied after a variable time delay $t_{d}$. This method can be used to study the time evolution of photogeneration. ${ }^{10}$

Figure 1 shows the quantum efficiency of photogeneration of holes $Y(E)$ in $5 \mu \mathrm{m} . \mathrm{H}_{2} \mathrm{Pc}$ single-layer films and two-layer films. The efficiency depends on the crystal structure: The efficiencies $Y(E)$ in $X$ - and $\tau-\mathrm{H}_{2} \mathrm{Pc}$ are high compared with those in $\alpha$ - and $\beta-\mathrm{H}_{2} \mathrm{Pc}$. According to the Onsager theory, the parameters $Y_{0}$ and $r_{0}$ in $X$ - and $\tau-\mathrm{H}_{2} \mathrm{Pc}$ are much larger than those of $\alpha-{ }^{-}$and $\beta-\mathrm{H}_{2} \mathrm{Pc}$. However, although $\alpha-\mathrm{H}_{2} \mathrm{Pc}$ exhibits a large carrier mobility, ${ }^{11}$ the photogeneration efficiency in $\alpha-\mathrm{H}_{2} \mathrm{Pc}$ is low compared with other polymorphic forms. In Fig. 2, the collected charge 


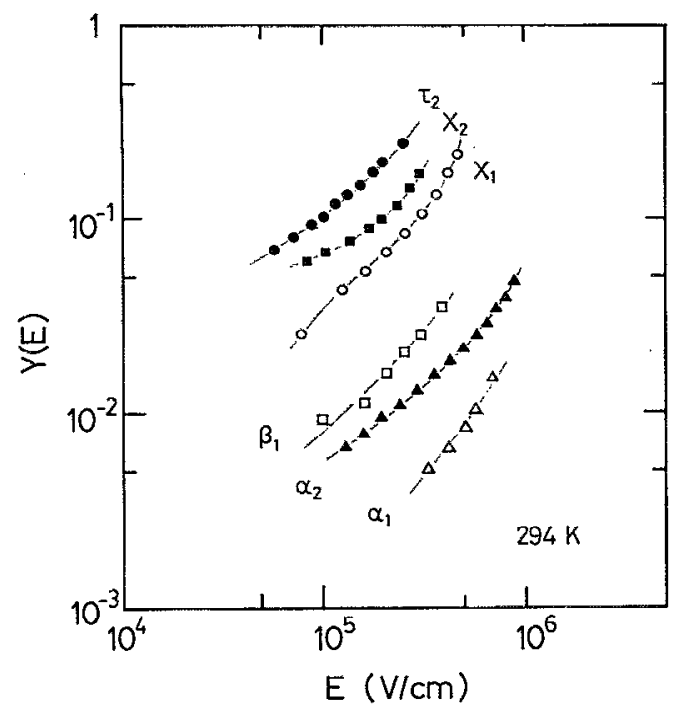

FIG. 1. Quantum efficiency of photogeneration of holes in metal-free phthalocyanines vs applied electric field. Greek letters show the crystal structure of phthalocyanines. The subscript numbers mean the sample structure: The subscript 1 denotes the single-layer structure and the subscript 2 denotes the two-layer structures.

signal $Q$ obtained from the DCF experiment is plotted as a function of delay time $t_{d}$. No significant decrease in $Q$ in $\beta-\mathrm{H}_{2} \mathrm{Pc}$ was observed within $100 \mathrm{~ms}$ time delay. The lifetime of a precursor state of free-charge carriers in $\alpha-\mathrm{H}_{2} \mathrm{Pc}$ is shorter than other polymorphic forms. Here, we ascribe the precursor state to the CT state due to the nearestneighbor electron transfer. It is regarded as the geminate charge pair separated by the nearest-neighbor intermolecular distance. ${ }^{8,9}$ The lifetime and structure of the initial CT state as well as the short-range carrier mobility play an important role in determining parameters of the Onsager theory, $Y_{0}$ and $r_{0}$.

The infrared (ir) absorption spectra of $\alpha-, \beta-, X$, and $\tau-\mathrm{H}_{2} \mathrm{Pc}$ dispersed in $\mathrm{KBr}$ were measured using an ir spectrometer in order to study the structural difference among the four $\mathrm{H}_{2} \mathrm{Pc}$ types. The $\mathrm{N}-\mathrm{H}$ stretching absorp-

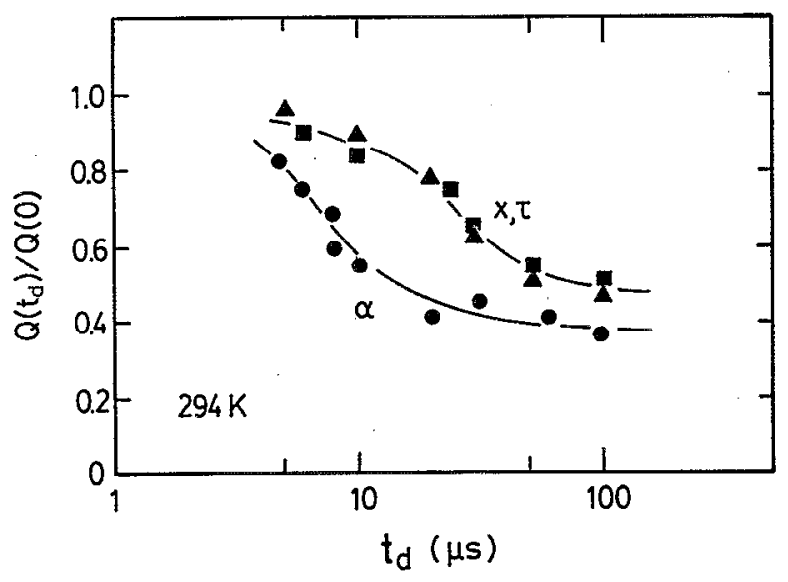

F1G. 2. Collected charge signals $Q$ in $\alpha-, X$-, and $\tau-\mathrm{H}_{2} \mathrm{Pc}$ vs delay time $t_{d}$.

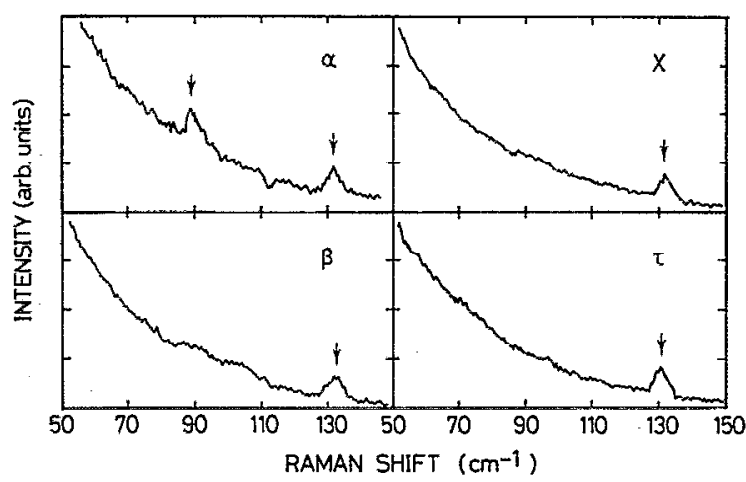

FIG. 3. Raman spectra of $\alpha-, \beta-, X-$, and $\tau-\mathrm{H}_{2} \mathrm{Pc}$ in a low wavenumber region. Note that $\alpha-\mathrm{H}_{2} \mathrm{Pc}$ has an additional Raman peak at $89 \mathrm{~cm}^{-1}$.

tion region near $3300 \mathrm{~cm}^{-1}$ and the $700-800 \mathrm{~cm}^{-1}$ region $^{12}$ were particularly sensitive for observing differences among the four crystal structures. The ir spectra of $X$ - and $\tau-\mathrm{H}_{2} \mathrm{Pc}$ were similar to those of $\alpha-\mathrm{H}_{2} \mathrm{Pc}$ near $3300 \mathrm{~cm}^{-1}$, but similar to that of $\beta-\mathrm{H}_{2} \mathrm{Pc}$ in the $700-800 \mathrm{~cm}^{-1}$ region: $X$ - and $\tau-\mathrm{H}_{2} \mathrm{Pc}$ are intermediate states between $\alpha$ - and $\beta-\mathrm{H}_{2} \mathrm{Pc}$. Furthermore, Fig. 3 shows Raman spectra of $\alpha$-, $\beta-, X$-, and $\tau-\mathrm{H}_{2} \mathrm{Pc}$ in a low wavenumber region. The intermolecular interaction and the stacking habit of molecules are considered to affect low-wavenumber Raman spectra. The sample powders were excited by a significantly attenuated $514.5 \mathrm{~nm}$ Ar laser $\left(\sim 1 \mathrm{~W} / \mathrm{cm}^{2}\right)^{13}$ in a vacuum under a quasibackscattering configuration. In $\alpha-\mathrm{H}_{2} \mathrm{Pc}$, two peaks were observed at 89 and $132 \mathrm{~cm}^{-1}$. On the other hand, in $\beta-, X$-, and $\tau-\mathrm{H}_{2} \mathrm{Pc}$, only the peak at 132 $\mathrm{cm}^{-1}$ was observed. At present, we cannot assign these peaks to characteristic vibrational modes. Raman spectra of $\alpha-\mathrm{H}_{2} \mathrm{Pc}$ significantly differ from those of other polymorphic forms. Furthermore, the time-resolved ir absorption experiment ${ }^{14}$ shows that the lifetime of triplet excitons in $\alpha-\mathrm{H}_{2} \mathrm{Pc}(10 \mu \mathrm{s})$ is shorter than those in $\beta$ - and $\tau-\mathrm{H}_{2} \mathrm{Pc}$ $(100 \mu \mathrm{s})$. It is therefore concluded that the stacking of molecules and electronic structure of $X$ - and $\tau-\mathrm{H}_{2} \mathrm{Pc}$ bear some resemblance to those of $\beta-\mathrm{H}_{2} \mathrm{Pc}$ rather than $\alpha-\mathrm{H}_{2} \mathrm{Pc}$.

Let us now consider the relationship between the stacking habit of $\mathrm{H}_{2} \mathrm{Pc}$ molecules and the photocarrier generation process. Normal projection of two parallel molecules is illustrated in Fig. 4. The electronic properties are related to the overlap between molecules. $\alpha-\mathrm{H}_{2} \mathrm{Pc}$ has a large intermolecular $\pi$ wavefunction overlap resulting in a large carrier mobility. In $\beta-\mathrm{H}_{2} \mathrm{Pc}$, on the other hand, the intermolecular hydrogen $(\mathrm{N}-\mathrm{H})$ bonding is formed and the intermolecular $\pi$ wavefunction overlap is small. ${ }^{15}$ Consequently, $\beta-\mathrm{H}_{2} \mathrm{Pc}$ exhibits a low carrier mobility. As mentioned above, the stacking of molecules in $X$ - and $\tau-\mathrm{H}_{2} \mathrm{Pc}$ is between that of $\alpha$ - and $\beta-\mathrm{H}_{2} \mathrm{Pc}$, and the structure of $X$ - and $\tau-\mathrm{H}_{2} \mathrm{Pc}$ bears some resemblance to that of $\beta-\mathrm{H}_{2} \mathrm{Pc}$. However, the photogeneration efficiencies in $X$ - and $\tau-\mathrm{H}_{2} \mathrm{Pc}$ are an order of magnitude higher than those in $\beta-\mathrm{H}_{2} \mathrm{Pc}$. These results imply that the photogeneration process in $\mathrm{H}_{2} \mathrm{Pc}$ is very sensitive to the degree of the intermolecular overlap of $\pi$ electron wavefunctions. Moreover, Sharp and Lardon ${ }^{12}$ 


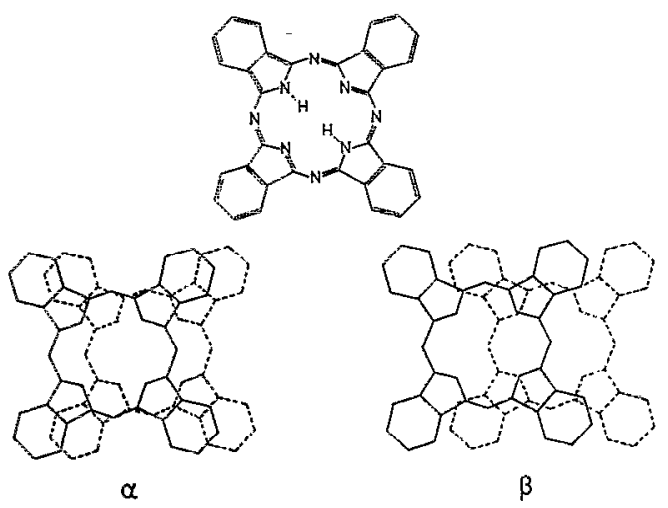

F1G. 4. Normal projection of two parallel molecules in $\alpha$ - and $\beta-\mathrm{H}_{2} \mathrm{Pc}$. Solid and dotted lines outline the upper and the underlying molecules, respectively.

proposed that $X-\mathrm{H}_{2} \mathrm{Pc}$ has a dimer structure. The initial charge separation due to the nearest-neighbor electron transfer is enhanced and the precursor $\mathrm{CT}$ state of free carriers is stable in the dimer structure of $X-\mathrm{H}_{2} \mathrm{Pc}$. Therefore, the initial yield $Y_{0}$ is very high. Since there is no significant difference in photoelectronic properties between $X$ - and $\tau-\mathrm{H}_{2} \mathrm{Pc}, \tau-\mathrm{H}_{2} \mathrm{Pc}$ may have a dimer structure like $X-\mathrm{H}_{2} \mathrm{Pc}$. We point out that the overlap of the intermolecular wavefunction and the structure of the nearest-neighbor separation charge pairs control the photocarrier generation in $\mathrm{H}_{2} \mathrm{Pc}$.

In conclusion, we have shown that the quantum effi- ciency of photocarrier generation in metal-free phthalocyanines is very sensitive to the stacking habit of metal-free phthalocyanine molecules. It is pointed out that the intermolecular overlap of the $\pi$ electron wavefunctions and the stacking habit of molecules control the photoconduction dynamics of $\mathrm{H}_{2} \mathrm{Pc}$. Organic photoconductors having a stable initial $\mathrm{CT}$ state due to the nearest-neighbor electron transfer are expected to exhibit high photogeneration efficiencies.

The authors would like to thank Y. Katsuya, C. Kato, and $\mathrm{Y}$. Endoh for fruitful discussions.

' R. O. Loutfy, Ah-M. Hor, C.-K. Hsiao, G. Baranyi, and P. Kazmaier, Pure Appl. Chem. 60, 1047 (1988).

${ }^{2}$ P. M. Borsenberger and D. S. Weiss, Handbook of Imaging Materials (Marcel Dekker, New York, 1990), Ch. 10.

${ }^{3}$ L. Onsager, Phys. Rev. 54, 554 (1938).

${ }^{4}$ D. M. Pai and R. C. Enck, Phys. Rev. B 11, 5163 (1975).

${ }^{5}$ S. Rackovsky and H. Scher, Phys. Rev. Lett. 52, 453 (1984).

${ }^{6}$ J. Mort and G. Pfister, Electronic Properties of Polymers, edited by J. Mort and G. Pfister (Wiley, New York, 1982), p. 215; D. M. Pai, Physics of Disordered Materials, edited by D. Adler, $\mathrm{H}$. Fritzsche, and $\mathrm{S}$. R. Ovshinsky (Plenum, New York, 1985), p. 579.

${ }^{7}$ J. Noolandi and K. M. Hong, J. Chem. Phys. 70, 3230 (1979).

${ }^{8}$ Z. D. Popovic, Chem. Phys. 86, 311 (1984).

${ }^{9}$ C. L. Braun, J. Chem. Phys. 80, 4157 (1984); T. E. Goliber and J. H. Perlstein, J. Chem. Phys. 80, 4162 (1984).

${ }^{10}$ J. Mort, M. Morgan, S. Grammatica, J. Noolandi, and K. M. Hong, Phys. Rev. Lett. 48, 1411 (1982).

${ }^{11}$ M. Pope and C. E. Swenberg, Electronic Processes in Organic Crystals, (Clarendon, Oxford, 1982), p. 659.

${ }_{12}$ J. H. Sharp and M. Lardon, J. Phys. Chem. 72, 3230 (1968).

${ }^{13}$ Under an intense laser excitation, $\beta-\mathrm{H}_{2} \mathrm{Pc}$ was transformed into $\alpha-\mathrm{H}_{2} \mathrm{Pc}$.

${ }^{14} \mathrm{C}$. Kato and Y. Katsuya (private communications).

${ }^{15}$ S. E. Harrison and J. M. Assour, J. Chem. Phys. 40, 365 (1964). 